\title{
A Relative Method of Video Coding using Different Wavelets with SPIHT
}

\author{
Nithin.S.S, L. Padma Suresh
}

\begin{abstract}
Lossy or lossless data compression is needed to Bring down the storage size and greater transmission rate. Wavelet transform is one of the ideal methods used for video compression. Different types of wavelet transforms are now available. In this paper, video is compressed by different wavelets and modified version of SPIHT. Totally seven types of wavelets are used here to compress the video, MSPIHT is used as encoding technique. They are (i) Video coding using duabechies wavelet and MSPIHT (VDM) (ii) Video coding using haar wavelet and MSPIHT (VHM) (iii) video coding using bi orthogonal wavelet and MSPIHT (VBM) (iv) video coding using symlet wavelet and MSPIHT (VSM) (v) video coding using coiflet wavelet and MSPIHT (VCM) (vi) video coding using demeyer wavelet and MSPIHT (VDMM) and (vii) video coding using Mexican hat wavelet and MSPIHT (VMHM). Then we calculate the PSNR and compression ratio for knowing the performance of the system.
\end{abstract}

Index Terms: (EWT) Empirical wavelet transforms, VHM, VDM, VBM, VSM, VCM, VDMM, VMHM and PSNR

\section{INTRODUCTION}

The ideas of digital image processing (DIP) have begun applications in different areas, such as astronomy, genetics, remote sensing, video communications, and medical. Image processing educations require a broad range of people from different educational backgrounds. Compression is an operation deliberate to provide a digital description of a signal. There are many applications that is only applicable when Image, video, and audio signals are in compressed form. Without compression, most of these applications are not usable. Video compression is valuable to any group that is used for viewing and storing of videos.

Wavelet transform is one of the accepted methods in video compression. Therefore here we use different types of wavelets for video compression. By minimizing the size of the data we can transmit additional amount of data. Mainly there are two different categories of compression methods: lossless and lossy compression. The major idea of compression is to diminish the size of the video without loss. In video compression, based on the quality of the data some losses may be admissible in reconstruction.

Currently multiple number of compression techniques are there, Wavelet had a immense outcome in the domain of compression and restoration. The main complication behind

\section{Revised Manuscript Received on September 25, 2019}

Mr.Nithin S.S, Research Scholar, Department of Electronics and Communication, Noorul Islam University, Tamil Nadu, India

Dr. L.Padma Suresh, Professor and Principal,Department of Computer Science, Baselios Mathews II College Of Engineering, Sasthamkotta.Kerala India the compression is the reconstruction of the original video. However, fragility is there in decomposition part while we are using wavelet.

Therefore to stay away from this case, new multi resolution techniques become introduced for more effective decomposition. Here seven different types of wavelets are used for decomposition then modified SPIHT is used as encoding technique. Figure 1 shows the schematic representation of basic compression.

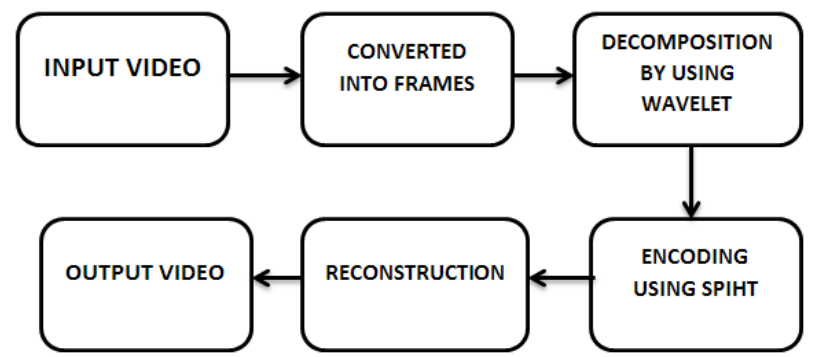

Figure 1: Schematic representation of basic compression

In general, the coefficients used in the wavelet transform are used to reproduce the original image excently. Here we are usinng seven different types of wavelets. They are duabechies, haar, bi orthogonal, symlet, coiflet, demeyer and Mexican hat wavelet.

\section{VIDEO CODING USING DUABECHIES WAVELET AND MSPIHT (VDM)}

Here we decompose the video using duabechies wavelet. Two properties are there in the duabechies wavelet, first property is that they have limited number of non-zero coefficients. Therefore dense support is there between scaling coefficients and wavelets. The second property is that first k-1 moment of the wavelets is nil. Duabechies wavelet varies from $\mathrm{db} 1$ to $\mathrm{db} 15$.The number represent the coefficients. Depending upon the vanishing moments the db number will varies In this method we compress the video using duabechies wavelet and modified SPIHT. Here, first we convert the video in to frames with the help of matlab. For decomposition we use duabechies wavelet. After this process we encode it by using modified SPIHT. Next we transmit and receive this data. For proper reconstruction we use inverse wavelet transform and decoder. The performance of our system is deliberated by PSNR and compression ratio. The results show that our system is good. Fig 2 shows the structure of this idea. 


\section{VIDEO CODING USING HAAR WAVELET AND MSPIHT (VHM)}

In this method the decomposition part is done by using haar wavelet. Haar wavelet is an uncomplicated method, so it is very easy to implement. In haar the frame is denoted as a matrix form which contains 0 to 255 values.

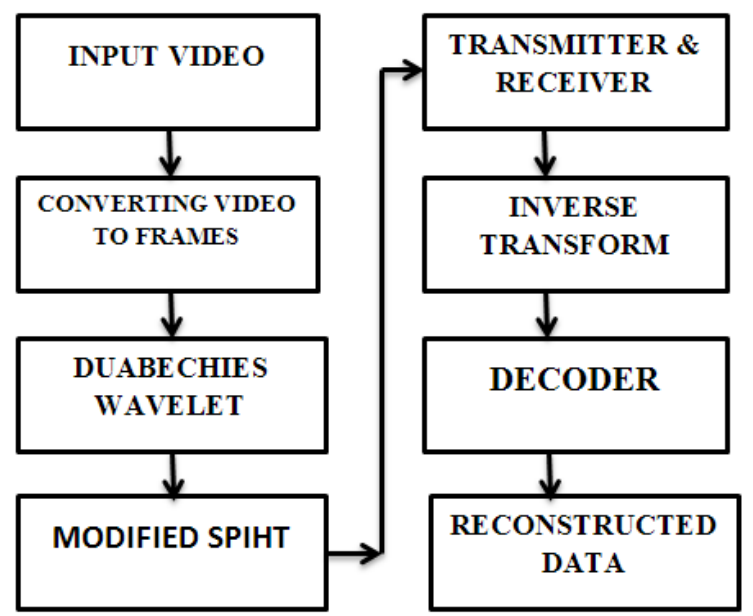

Figure 2: Block diagram of video coding using duabechies wavelet and modified SPIHT.

We will take average and difference for transmitting string of data. By using this operation a new matrix is formed. Then we repeatly were doing this operation for getting the final results. After doing certain steps we will get the final output. In this method we compress the video using haar wavelet and modified SPIHT. Here, first we convert the video in to frames with the support of matlab. For decomposition we use haar wavelet. After this process we encode it by using modified SPIHT. After reducing the size of the video, we transmit and receive this data. For proper reconstruction we use inverse wavelet transform and decoder. The performance of our system is analyzed by PSNR and compression ratio. The results show that our system got better results. Fig 3 represents the structure of this idea.

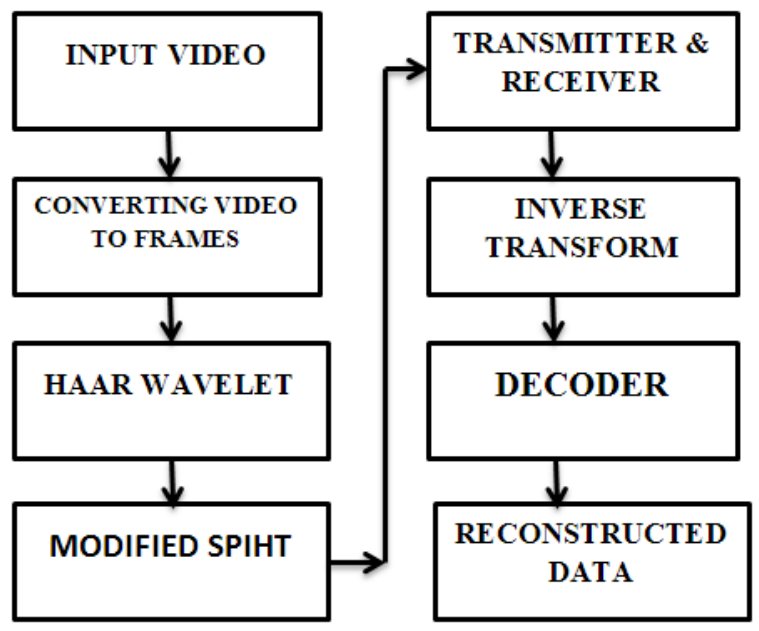

Figure 3: Block diagram of video coding using haar wavelet and modified SPIHT.

\section{VIDEO CODING USING BIORTHOG-ONAL WAVELET AND MSPIHT (VBM)}

Biorthogonal wavelet is related to haar wavelet. Here we use two wavelets, one of them is used for decomposition and the another one is used for better reconstruction. Here we are using two wavelets rather than single one. For analysing and synthesising two types of filters are used in biorthogonal wavelet. In this method we use biorthogonal wavelet for decomposition. In this method we compress the video using biorthogonal wavelet and modified SPIHT. Here, first we convert the video in to frames with the support of matlab. For the decomposition purpose we use biorthogonal wavelet. After this process we encode it by using modified SPIHT. After reducing the size of the video, we transmit and receive this data. For proper reconstruction we use inverse wavelet transform and decoder. The performance of our system is analyzed by PSNR and compression ratio CR. The results show that our system got better results. Figure 4 represents the structure of this idea.

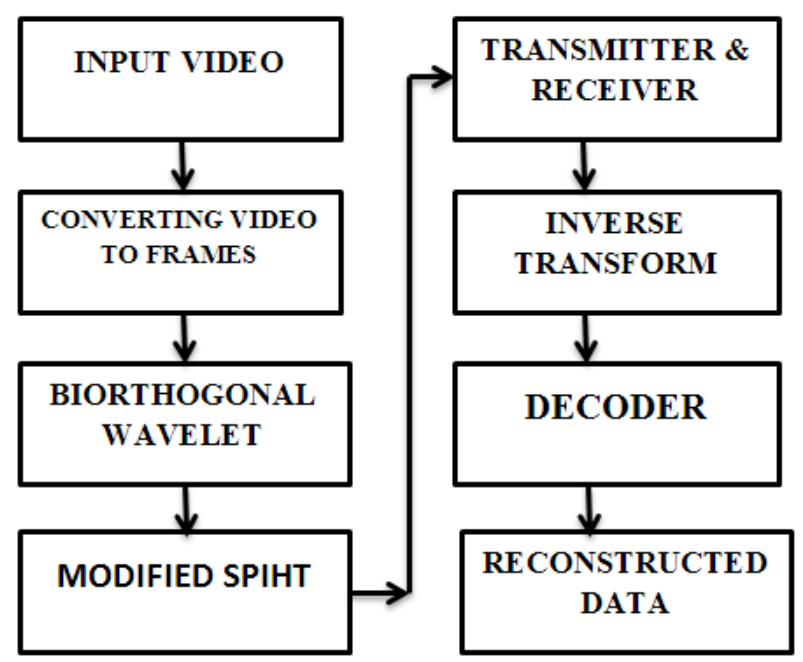

Figure 4: Block diagram of video coding using biorthogonal wavelet and modified SPIHT.

\section{VIDEO CODING USING SYMLET WAVELET AND MSPIHT (VSM)}

In this symlet wavelet is used for decomposition. The wavelet operations of the Daubechies wavelets are long away from equality since as Daubechies db wavelets select the smallest phase square root such that the strength focuses close the begining point of their assist, but Symlets pick each other position of roots to have nearer equality with linear complicated phase. Symlets are founded by Daubechies as updation of the $\mathrm{Db}$ family, except equality, rests other properties of Daubechies db and Symlet group are almost same.

In this approach, we compress the video using symlet wavelet and modified SPIHT. Here, first we convert the video in to frames with the use of matlab. For decomposition we use symlet wavelet. After this process we encode it by using

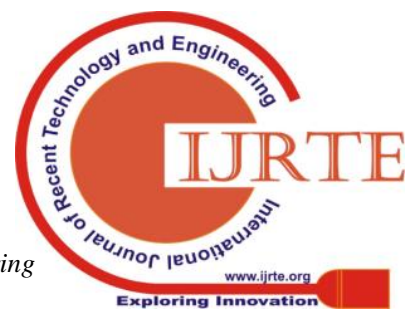


modified SPIHT. After reducing the size of the video, we transmit and receive this data. For proper decompression of the original video we use inverse wavelet transform and decoder. The performance of our system is analysed by PSNR and compression ratio. The results show that our system got better results. Fig 5 denote the block diagram of this method.

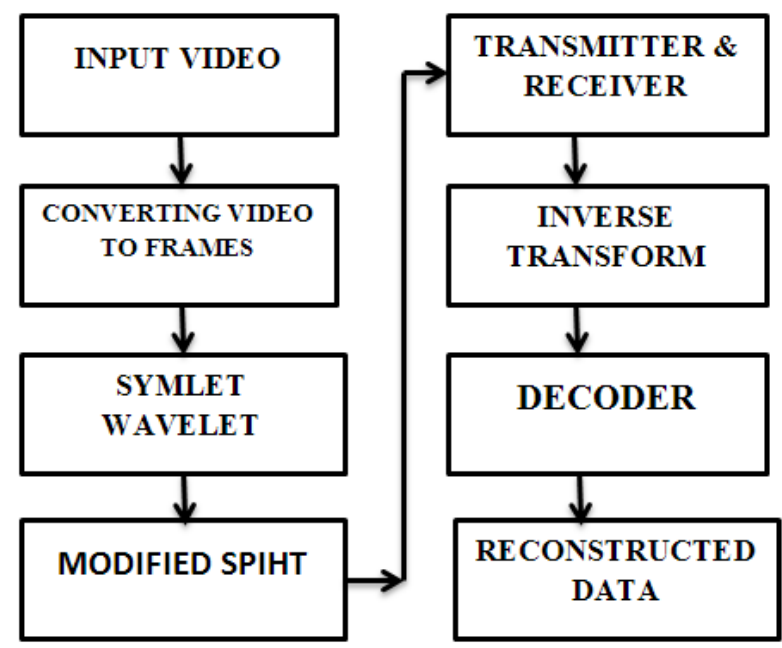

Figure 5: Block diagram of video coding using symlet wavelet and modified SPIHT.

\section{VIDEO CODING USING COIFLET WAVELET AND MSPIHT (VCM)}

For decomposition here we use coiflet wavelet. This wavelet is designed by Daubechies at an appeal of R. Coif man. This behaviour has $2 \mathrm{~N}$ bits equivalent to 0 and the scaling behaviour has $2 \mathrm{~N}-1$ bits corresponding to 0 . These two behaviours have a carry of span $6 \mathrm{~N}-1$. Closely supported wavelets with longest number of vanishing bits for phi and psi for a given appropriate range.



Figure 6: Block diagram of video coding using coiflet wavelet and modified SPIHT.

In this method of approach, we compress the video using coiflet wavelet and modified SPIHT. Here, first we convert the video in to frames with the use of matlab. For decomposition purpose we are using symlet wavelet. After this step we encode the decomposed frames by using modified version of SPIHT. After minimising the size of the video, we transmit and receive this data. For appropriate decompression of the original video we use inverse wavelet transform and decoder. To analyse the performance of our system PSNR and compression ratio are calculated. The results show that our system got better results. Fig 6 represents the block diagram of the topic.

\section{VIDEO CODING USING DEMEYER WAVELET AND MSPIHT (VDMM)}

This For decomposition here we use demeyer wavelet. The scaling operation and wavelet can fit to the space and can reduce quicker than the others. The wavelet and the scaling operation are fabricated in the frequency discipline with the help of trigonometry. The scaling function is uniform at the point zero, while the wavelets are uniform around point 1/2. This wavelet is a differentiable property.

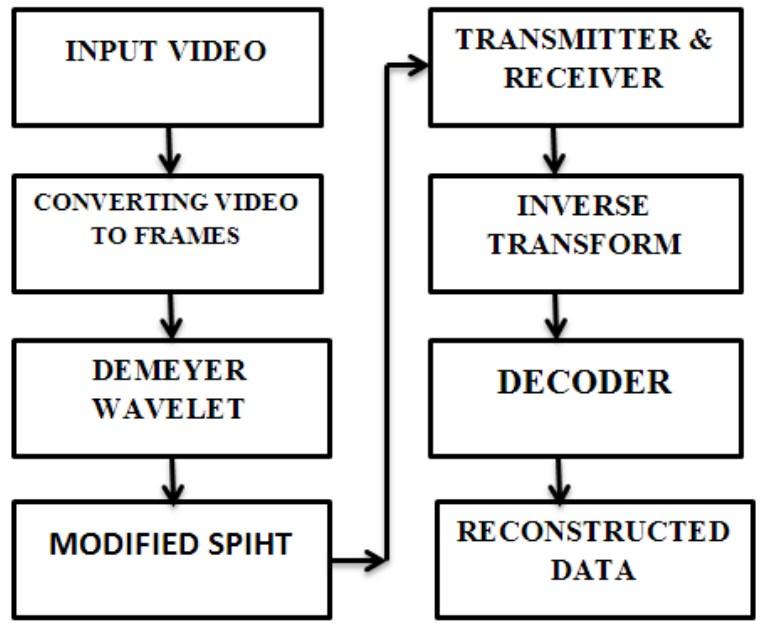

Figure 7: Block diagram of video coding using demeyer wavelet and modified SPIHT.

In this method we compress the video using demeyer wavelet and modified SPIHT. Here, first we convert the video in to frames with the support of matlab. For decomposition we use demeyer wavelet. After this process we encode it by using modified SPIHT. After reducing the size of the video, we transmit and receive this data. For proper reconstruction we use inverse wavelet transform and decoder. The performance of our system is analysed by PSNR and compression ratio. The results show that our system got better results. Fig 7 represents the block diagram of this concept.

\section{VIDEO CODING USING MEXICAN HAT WAVELET AND MSPIHT. (VMHM)}

This is the last method used here in video compression. In this type mexican hat wavelet is used in decomposition technique. In this type of approach, we compress the video using mexican hat wavelet and modified SPIHT. Here, first we convert the video in to frames with the use of matlab. For decomposition we use

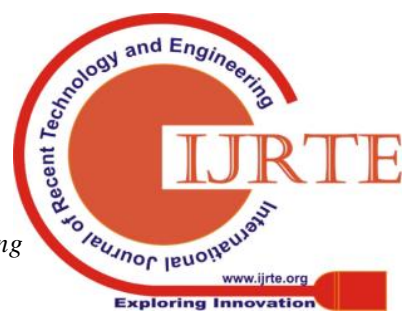


mexican hat wavelet. After this process we encode it by using modified SPIHT. After reducing the size of the video, we transmit and receive this data. For proper decompression of the original video we use inverse wavelet transform and decoder. The performance of our system is analysed by PSNR and compression ratio. The results show that our system got better results. Fig 8 shows the block diagram of the above system.

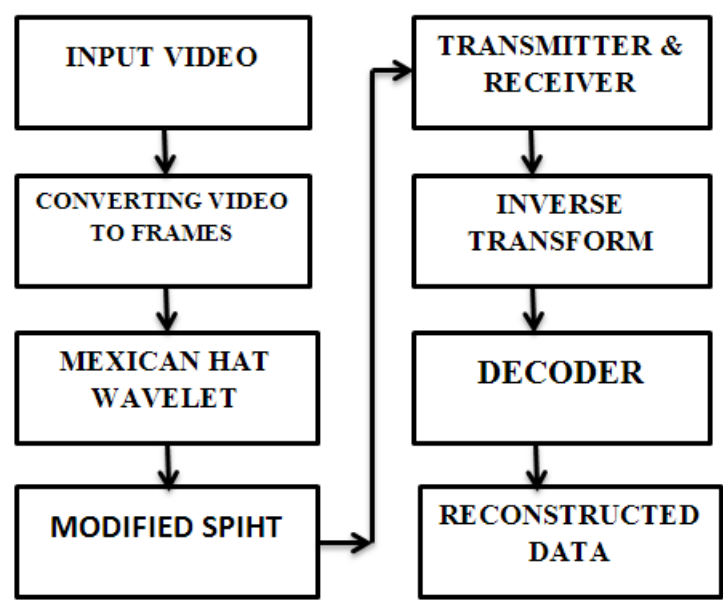

Figure 8: Block diagram of video coding using mexican hat wavelet and modified SPIHT.

\section{PERFORMANCE COMPARISON AND RESULTS}

This Here we analyze seven different types of video coding. By reducing the size transmission and storage become more comfortable. Figure 9 shows the performance analysis of different methods. In this research, we compress the video using seven different methods of wavelet. They are (i) Video coding using duabechies wavelet and MSPIHT (VDM) (ii) Video coding using haar wavelet and MSPIHT (VHM) (iii) video coding using bi orthogonal wavelet and MSPIHT (VBM) (iv) video coding using symlet wavelet and MSPIHT (VSM) (v) video coding using coiflet wavelet and MSPIHT (VCM) (vi) video coding using demeyer wavelet and MSPIHT (VDMM) and (vii) video coding using Mexican hat wavelet and MSPIHT (VMHM).

In my research, we implement different types of wavelets. In the seven different types of coding first we convert the video in to different frames. Then decompose these frames by using wavelet transform. Here I am using seven different types of wavelets. Then the performance is calculated by measuring PSNR and compression ratio. For encoding purpose, here I use SPIHT. The Mexican hat wavelet shows better PSNR and CR than the other methods. Figure 9 represents the performance analysis of different wavelets.
PSNR
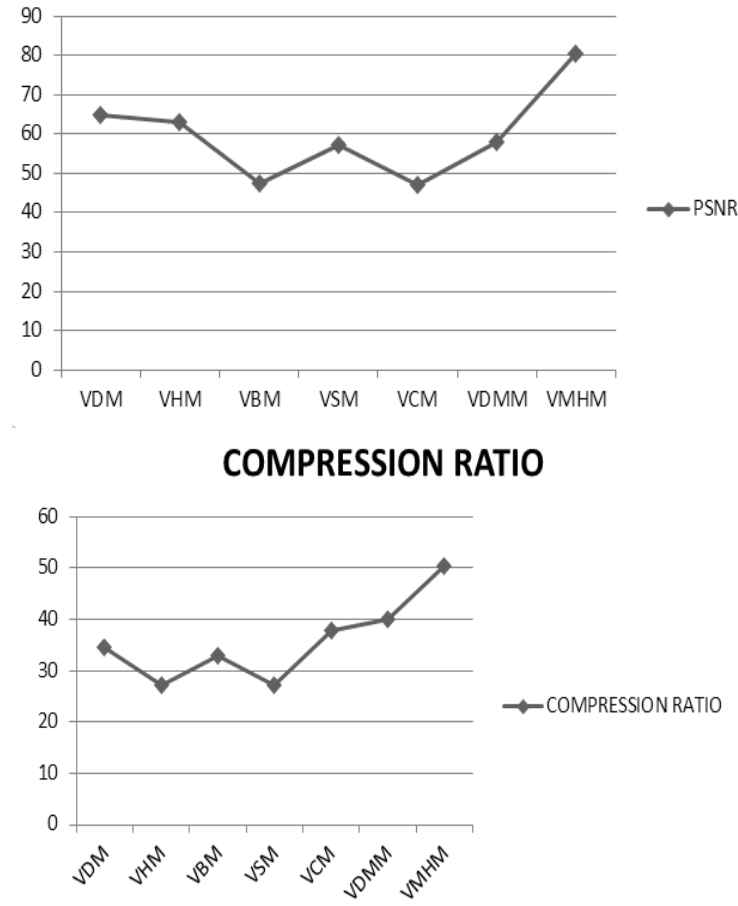

Figure 9: Performance analysis of different compression methods

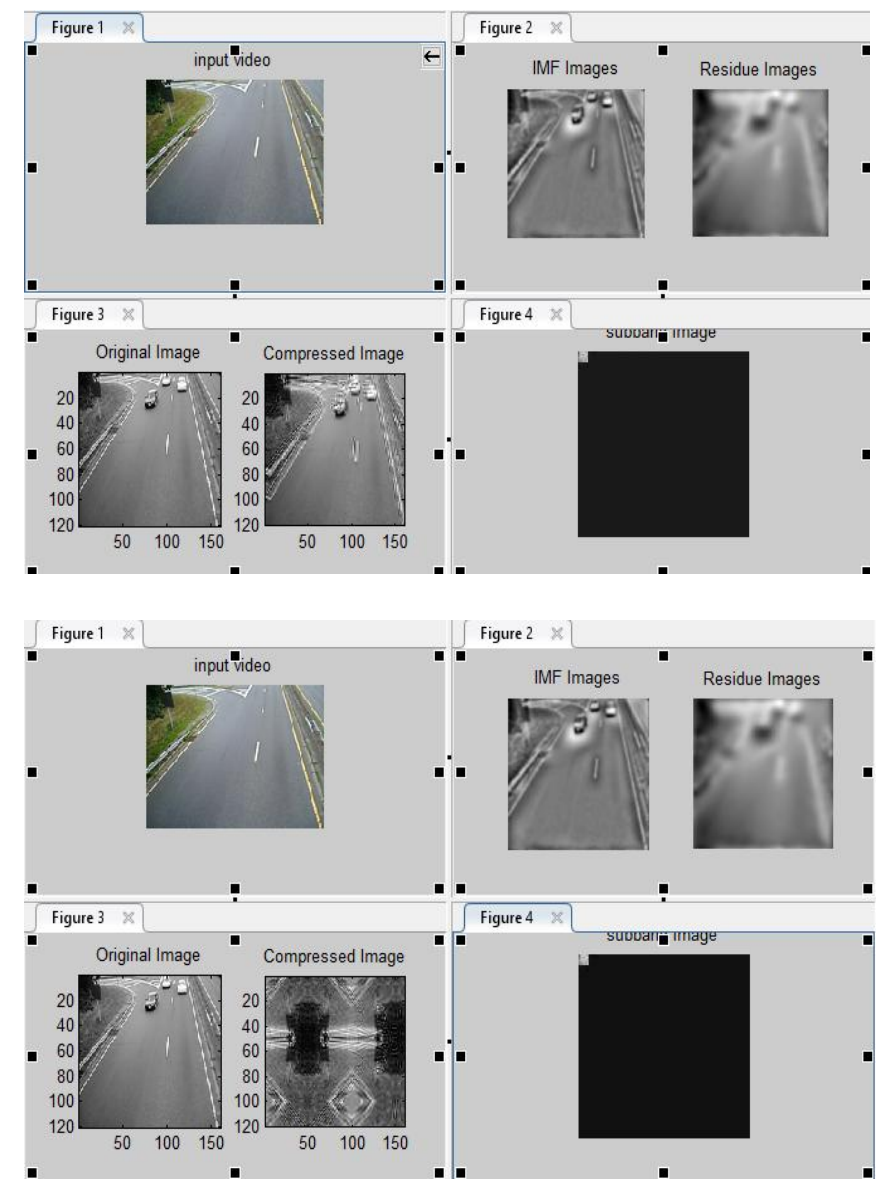

Figure 10: Results of haar wavelet and mexican hat wavelet 
Figure 10 represents the output of haar wavelet and Mexican hat wavelet. I think it is also a better technique for reducing the size of the video. In visual communication and broadcasting video compression is an essential task.

\section{CONCLUSION}

There are lot of methods are available in the market for compressing the video. Here I also analyse different types of wavelet compresion methods. We compress the video by using duabechies, haar, biorthogonal symlet, coiflet, demeyer and mexican hat wavelet. By comparing the PSNR and compression ratio video coding using mexican hat wavelet and MSPIHT got better results than other methods.

\section{REFERENCES}

[1] Daubechies, W. Sweldens, 1998, "Factoring wavelet into lifting steps", J. Fourier Anal. Appl., Vol.4(3), pp.247-269.

[2] IyyapanDhasarathan, VimalRathinasamy, Tang Chi, "Wavelet Based SPIHT Compression for DICOM Images", Linnaeus University, School of Computer Science, Physics and Mathematics, 2011.

[3] Madhuri A., "Digital Image Processing", an Algorithmic Approach", PHI, New Delhi, pp. 175-217, 2006.

[4] Swapna Devi, Niveditta "A New Method for Color Image Quality Assessment", International Journal of Computer Applications , Volume 15No.2, February 2011

[5] Wavelets and different multi resolution techniques.

[6] Woods R. E., Gonzalez R. C Digital Image Processing, Second Edition, ISBN: 0-20-118075-8

\section{AUTHORS PROFILE}

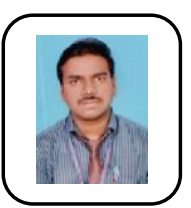

Mr.Nithin S.S,

Educational Profile:

Doing Ph.D- Noorul Islam University

ME-Communication System

BE-Electronics and Communication Engineering,

Teaching Experience: 8 Years

Area of Specialization:Image Processing, Published various

papers

Dr. L Padma Suresh.

received his B.E. Electrical and Electronics Engineering from Noorul Islam College of Engineering on 1999 and

M.E. Process Control and Instrumentation in 2000 from Annamalai University and Ph.D. Electrical Engineering in 2010 from Dr.M.G.R University and Ph.D Computer Science Engineering from M.S. University. He has 18years of diverse experience in teaching, Life member of ISTE, and Senior IEEE Member. His Research interests in Artificial Intelligence, Control Systems, Power Electronics and Image Processing. 